\title{
SNP-SIG Meeting 2011: Identification and annotation of SNPs in the context of structure, function, and disease
}

\author{
Yana Bromberg ${ }^{1 *}$, Emidio Capriotti ${ }^{2 *}$ \\ From SNP-SIG 2011: Identification and annotation of SNPs in the context of structure, function and disease \\ Vienna, Austria. 15 July 2011
}

\section{Overview}

Advances in high-throughput sequencing, genotyping, and characterization of haplotype diversity are consistently generating vast amounts of genomic data. Single Nucleotide Polymorphisms (SNPs) are the most common type of genetic variation. In the recent years the number of known SNPs has been increasing exponentially; the last release of the NCBI's dbSNP database contained more than 50 million human SNPs. SNPs are interesting as both markers of evolutionary history and in the context of their phenotypic manifestations (e.g. characteristic traits and diseases). For some diseases, e.g. sickle-cell anemia, the causative SNPs are well documented. In most other cases, the detection of disease-causing variants is still a problem. The genome-wide association studies (GWAS) provide insight into SNP-disease relationships. However, GWAS analysis is both experimentally and computationally expensive and fails to properly consider the rare variants, i.e. individual-specific SNPs that have yet to be documented on a population scale. This discrepancy between the deluge of SNP data and the lack of its interpretation spurs the development of the SNP impact annotation/prediction algorithms. In the near future, the study of genetic variation in disease and treatment options will be key for the development of the field of personalized medicine.

In 2010, the first edition of the Critical Assessment of Genomic Interpretation (CAGI; Berkeley, California) was organized to evaluate the ability of available computational

\footnotetext{
* Correspondence: YanaB@rci.rutgers.edu; emidio.capriotti@uab.es 'Department of Biochemistry and Microbiology, School of Environmental and Biological Sciences, Rutgers University, New Brunswick 76 Lipman Drive, NJ 08901, USA

2Department of Mathematics and Computer Science, University of Balearic Islands, Ctra. de Valldemossa Km 7.5, Palma de Mallorca, 07122 Spain Full list of author information is available at the end of the article
}

methods to predict the phenotypic impacts of genomic variation. Annotation of SNPs was also a hot topic in many other meetings, such as AIMM at ECCB 2010 (Ghent, Belgium), the HGVS 2010 meeting (Washington, DC) and PSB 2011 (Big Island of Hawaii, USA). In line with the increasing interest in the genetic variation analysis and annotation, on July $15^{\text {th }}, 2011$ we organized the first SNP Special Interesting Group (SNP-SIG) meeting at ISMB/ECCB'2011 in Vienna, Austria (http://snps.uib.es/ snp-sig/2011). This meeting attempted to summarize the field's research advances in the directions of "Annotation and prediction of structural/functional impacts of coding SNPs" and "SNPs and Personal Genomics: GWAS, populations and phylogenetic analysis". Over 70 scientists actively working in the field and strongly interested in its development have officially registered for the SIG. On the date of the meeting, an even larger number of ISMB participants have gathered to discuss their work, the state of the art, and future perspectives. In all, 17 presentation proposals and 13 posters were submitted to the SIG and eight works where selected for an oral presentation at the meeting.

Distinguished scientists were invited to share their visions of the field past, present, and future: Steven Brenner (University of California at Berkeley), Atul Butte (Stanford University), John Moult (University of Maryland, College Park), Burkhard Rost (Techinal University of Munich) and Mauno Vihinen (Lund University). A round table discussion on the most timely and important problems of SNP annotation was held, directed by Christopher Baker (University of New Brunswick), Maricel Kann (University of Maryland, Baltimore), Sean Mooney (Buck Institute), Pauline Ng (Genome Institute of Singapore) and Mauno Vihinen (Lund University).

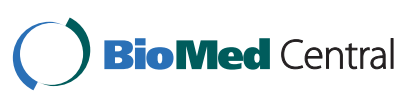


We have invited all SIG presenters to submit full research papers for publication in this special issue of BMC Genomics. We adopted a peer review process to select ten exceptional works. The articles cover different aspects of the field, presenting databases and tools for the annotation of SNPs as well as novel scientific advances achieved based on these resources. The described methods use different types of information derived from sequence, evolution, function and structure to analyze large sets of variations. They address SNP-associated (1) specific protein function [1] or (2) structure/stability $[2,3]$ changes or focus on (3) non-coding SNPs [4,5] or (4) specific disease classes [6,7]. Presented work gives new life to the information buried in literary free text [8] and outlines the potential of using SNP functional impacts to predict disease involvement [9]. We also include a method developer tutorial/framework that will be very helpful for all future work in the field [10].

\section{Next meeting}

We are now working on the organization of the next edition of the SNP-SIG meeting to be held in the context of the ISMB 2012, Long Beach, California. Further information about the SNP-SIG 2012 is available on our web site (http://snps.uib.es/snp-sig). Submissions of posters and presentation proposals are welcome.

\section{Competing interests}

The authors were organizers and co-chairs of the SNP-SIG meeting, and declare they have no other conflict of interest.

\footnotetext{
Acknowledgments

We would like to acknowledge Janita Thusberg (Buck Institute) for helping us with the poster session. We thank Frank Schacherer and BIOBASE International for the presentation of the HGMD database and for the financial support of the meeting. We are very grateful for the support of the ISMB steering committee, especially Burkhard Rost (Technical University of Munich), Michal Linial (Hebrew University), and Hershel Safer (Tel Aviv University). We would like to extend special thanks for all help to the ISMB organizational committee and specifically Steven Leard and Jeremy Hennig. Also, we thank Isobel Peters and Sarah Headley from the editorial staff of BioMed Central for their help with releasing this issue. Finally, we are especially grateful for the patience and help of our colleagues around the world who reviewed the abstracts and submitted manuscripts. The SIG meeting or this issue would not be possible without them:

Leonardo Arbiza (Cornell University), Christopher Baker (University of New Brunswick), Dario Boffelli (University of California, Merced), William S. Bush (Vanderbilt University), Adrien Coulet (University of Lorraine), Rong Chen (Stanford University), Jianlin Cheng (University of Missouri, Columbia), Xavier de la Cruz (Institut de Biologia Molecular de Barcelona), Tjaart de Beer (European Bioinformatics Institute), Hernán Dopazo (Centro de Investigacion Principe Felipe), Andre Franke (Christian-Albrechts-University of Kiel), Iddo Friedberg (Miami University), Liang-Tsung Huang (Mingdao University), Andrew Johnson (National Institutes of Health, US), Jae-Yoon Jung (Harvard University), Peter Kang (Stanford University), Adam Kowalczyk (University of Melbourne), Vidhya Krishnan (Genome Institute of Singapore), Phil Hyoun Lee (Massachusetts General Hospital), Jing Li (Case Western Reserve University), Marc A. Marti-Renom (National Center for Genomic Analysis, Spain), Sean Mooney (Buck Institute), Matthew Mort (Cardiff University), Alessio Naccarati (Institute of Experimental Medicine, Czech Republic), Pauline Ng (Genome Institute of Singapore), Yanay Ofran (Bar Ilan University),
} Gaurav Pandey (Mount Sinai School of Medicine), Yue Peng (Genentech Inc.),
Predrag Radivojac (University of Indiana, Bloomington), Lipika Ray (University of Maryland, Rockville), Susanna Repo (European Bioinformatics Institute), Joke Reumers (Katholieke Universiteit Leuven), Joost Schymkowitz (Katholieke Universiteit Leuven), Paul D. Thomas (University of Southern California, Los Angeles), Janita Thusberg (Buck Institute), Ali Torkmani (The Scripps Research Institute), Dennis P. Wall (Harvard University), Zemin Zhang (Genentech Inc.), Yiqiang Zhao (Buck Institute).

This article has been published as part of BMC Genomics Volume 13 Supplement 4, 2012: SNP-SIG 2011: Identification and annotation of SNPs in the context of structure, function and disease. The full contents of the supplement are available online at http://www.biomedcentral.com/ bmcgenomics/supplements/13/S4.

\section{Author details}

${ }^{1}$ Department of Biochemistry and Microbiology, School of Environmental and Biological Sciences, Rutgers University, New Brunswick 76 Lipman Drive, NJ 08901, USA. 'Department of Mathematics and Computer Science, University of Balearic Islands, Ctra. de Valldemossa Km 7.5, Palma de Mallorca, 07122 Spain.

Published: 18 June 2012

\section{References}

1. Izarzugaza, del Pozo, Vazquez, Valencia : Prioritization of pathogenic mutations in the protein kinase superfamily. BMC Genomics 2012, 13(Suppl 4):3.

2. Shaefer, Rost : Predict impact of single amino acid change upon protein structure. BMC Genomics 2012, 13(Suppl 4):4.

3. Juritz, Fornasari, Martelli, Fariselli, Casadio, Parisi : On the effect of protein conformation diversity in discriminating among neutral and disease related single amino acid substitutions. BMC Genomics 2012, 13(Suppl 4):5.

4. Ritz, Martin, Laederach : Evaluating our ability to predict the structural disruption of RNA by SNPs. BMC Genomics 2012, 13(Suppl 4):6.

5. Riva : Large-scale computational identification of regulatory SNPs with rSNP-MAPPER. BMC Genomics 2012, 13(Suppl 4):7.

6. Martelli, Fariselli, Balzani, Casadio : Predicting cancer-associated germline variations in proteins. BMC Genomics 2012, 13(Suppl 4):8

7. Nehrt, Peterson, Park, Kann: Domain landscapes of somatic mutations in cancer. BMC Genomics 2012, 13(Suppl 4):9.

8. Naderi, Witte : Automated extraction and semantic analysis of mutation impacts from the biomedical literature. BMC Genomics 2012, 13(Suppl 4):10.

9. Schaefer, Bromberg, Achten, Rost: Disease-related mutations predicted to impact protein function. BMC Genomics 2012, 13(Suppl 4):11.

10. Vihinen: How to evaluate performance of prediction methods? Measures and their interpretation in variation effect analysis. BMC Genomics 2012, 13(Suppl 4):2.

doi:10.1186/1471-2164-13-S4-S1

Cite this article as: Bromberg and Capriotti: SNP-SIG Meeting 2011: Identification and annotation of SNPs in the context of structure, function, and disease. BMC Genomics 2012 13(Suppl 4):S1.

\section{Submit your next manuscript to BioMed Central and take full advantage of:}

- Convenient online submission

- Thorough peer review

- No space constraints or color figure charges

- Immediate publication on acceptance

- Inclusion in PubMed, CAS, Scopus and Google Scholar

- Research which is freely available for redistribution 